\title{
RNA sequencing analysis of FGF2-responsive transcriptome in skin fibroblasts
}

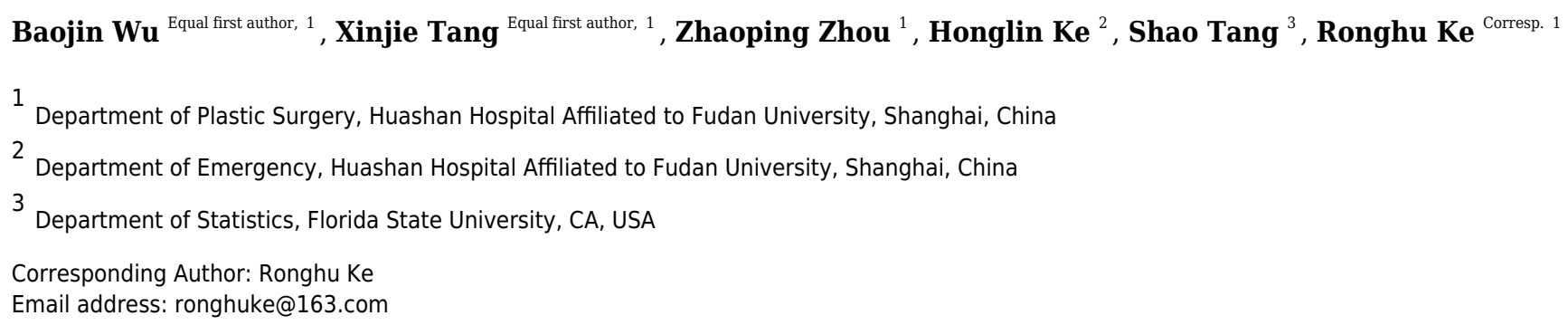

Background: Fibroblast growth factor 2 (FGF2) is a highly pleiotropic cytokine with antifibrotic activity in wound healing. During the process of wound healing and fibrosis, fibroblasts are the key players. Although accumulating evidence has suggested the antagonistic effects of FGF2 in the activation process of fibroblasts, the mechanisms by which FGF2 hinders the fibroblast activation remains incompletely understood. This study aimed to identify the key genes and their regulatory networks in skin fibroblasts treated with FGF2. Methods: RNA-seq was performed to identify the differentially expressed mRNA (DEGs) and IncRNA between FGF2-treated fibroblasts and control. DEGs were analyzed by Gene Ontology (GO) and Kyoto Encyclopedia of Genes and Genomes (KEGG). Furthermore, the networks between mRNAs and IncRNAs were constructed by Pearson correlation analysis and the networkanalyst website. Finally, hub genes were validated by real time-PCR. Results: Between FGF2-treated fibroblasts and control fibroblasts, a total of 1475 DEGs was obtained. These DEGs were mainly enriched in functions such as the ECM organization, cell adhesion, and cell migration. And they were mainly involved in ECMreceptor interaction, PI3K-Akt signaling, and the Hippo pathway. The hub DEGs included COL3A1, COL4A1, LOX, PDGFA, TGFBI, and ITGA10. Subsequent real-time PCR, as well as bioinformatics analysis consistently demonstrated that the expression of ITGA10 was significantly upregulated while the other 5 DEGs (COL3A1, COL4A1, LOX, PDGFA, TGFBI) were downregulated in FGF2-treated fibroblasts. Meanwhile, 213 differentially expressed IncRNAs were identified and three key IncRNAs (HOXA-AS2, H19, and SNHG8) were highlighted in FGF2-treated fibroblasts. Conclusion: The current study comprehensively analyzed the FGF2-responsive transcriptional profile and provided candidate mechanisms that may account for FGF2-mediated wound healing. <!--[if !supportLineBreakNewLine]--> $<$---[endif]--> 
1

2 Title: RNA sequencing analysis of FGF2-responsive transcriptome in skin fibroblasts

3

4 Authors: Baojin $\mathrm{Wu}^{1 *}$; Xinjie Tang ${ }^{1 \#}$; Zhaoping Zhou ${ }^{1}$; Honglin $\mathrm{Ke}^{\mathbf{2}}$; Shao Tang ${ }^{3}$, Ronghu Ke 5

6

7 Affiliation: ${ }^{1}$ Department of Plastic Surgery, ${ }^{2}$ Department of Emergency, Huashan Hospital 8 Affiliated to Fudan University, Shanghai, China. ${ }^{3}$ Department of Statistics, Florida State 9 University, FL, USA.

10

11 \#Baojin $\mathrm{Wu}$ and Xinjie Tang are equal to this work.

12

\section{\$Correspondence to:}

Ronghu Ke, MD

Department of Plastic Surgery, Huashan Hospital, Fudan University, Shanghai, China

No. 12, Wu Lu Mu Qi Road (M), Shanghai 200040, China.

Tel: +86(021) 52887832

Email: ronghuke@163.com 


\section{Abstract}

Background: Fibroblast growth factor 2 (FGF2) is a highly pleiotropic cytokine with antifibrotic activity in wound healing. During the process of wound healing and fibrosis, fibroblasts are the key players. Although accumulating evidence has suggested the antagonistic effects of FGF2 in

Keywords: FGF2, RNA-seq, fibroblast, wound healing 
Fibrosis is a fundamental wound healing process that occurs in almost every organ including lung, liver, kidney, heart, or skin. Despite numerous crucial differences among fibrotic pathologies of various organs, one of the commonalities among these affected organs is the activation and transdifferentiation of quiescent fibroblasts into contractile myofibroblasts (Yazdani et al. 2017). During the process of fibrosis, fibroblasts and myofibroblasts are the main effectors. Compared to non-activated fibroblasts, myofibroblasts are larger in the area and have their structural characterization with the presence of actin filament bundles containing alphasmooth muscle actin ( $\alpha$-SMA), which augments its ability to generate contractile force in the wound site. Myofibroblasts are also characterized as producing excessive extracellular matrix (ECM), particularly type I collagen and the fibronectin-extra domain A (EDA) isoform. Soon after their injury, local fibroblasts translate into the core of the wound and differentiate into contractile myofibroblasts, leading to wound retraction and healing. After closing the wound, myofibroblasts undergo apoptosis to clear the wound site (Vallee \& Lecarpentier 2019). However, under pathological conditions, persistent myofibroblasts activation results in an overproduction of collagen and the formation of the pathological scar. Thus, fibroblast differentiation into myofibroblasts is the key process in dysregulated wound healing as well as organ fibrosis. Intervening into myofibroblast-induced pro-fibrotic activities using drug targeting technologies can be a promising approach for developing novel therapeutics against fibrosis (Yazdani et al. 2017).

Fibroblast growth factor 2 (FGF2), also known as a basic fibroblast growth factor (bFGF), is one of the family members of mammalian fibroblast growth factors. FGF2 has low (18-kDa) and high (22-, 22.5-, 24-, and 34-kDa) molecular weight isoforms, which are translated from a single transcript by starting from alternative, in-frame start codons. These isoforms act predominantly in an autocrine or paracrine manner via the fibroblast growth factor receptors (FGFRs), which contains two receptor isoforms (IIIb or IIIc). FGF2 preferentially activates FGFR1c, FGFR3c and FGFR4 and shows some affinity to FGFR1b and FGFR2c (Ornitz et al. 1996). When FGF2 activates its receptor, intracellular adaptor and effectors proteins are recruited to stimulate the 
85

86

87

signal pathways, most notably the mitogen-activated protein kinases (MAPKs) and Akt/mTOR. By the canonical pathways, FGF2 was well studied especially in the field of ontogenesis, stem cell self-renewal, and tissue repair (Akl et al. 2016). As for FGF2 roles in tissue repair, enormous clinical applications particularly in China and to an extent in Japan, have been carried out. Based on the clinical research, FGF2 has been shown to have anti-fibrotic effects in conditions as diverse as burns, chronic wounds, oral ulcers, vascular ulcers, diabetic ulcers, pressure ulcers, and surgical incisions (Nunes et al. 2016) (Akita et al. 2008; Matsumine 2015; Ono et al. 2007). Moreover, FGF2 antagonized TGF $\beta 1$-induced differentiation of fibroblasts and thus affected fibrosis during wound repair (Dolivo et al. 2017a). Importantly, FGF2 was observed to induce a shift in gene expression to a more anti-fibrotic signature attenuated the expression of pro-fibrotic genes, including collagen I, collagen III, and $\alpha$-SMA (Dolivo et al. 2017b). Despite the extensive observations, the mechanisms by which FGF2 regulates the fibrotic response remain incompletely understood.

Microarray and high-throughput sequencing technologies are powerful tools that can be used to investigate potential target genes for diseases and underlying pathological mechanisms (Mery et al. 2019). By microarray technology, the expression profiles have been investigated in FGF2treated fibroblasts (Hernandez \& Dominko 2016; Kashpur et al. 2013). In these studies, high throughput transcriptional datasets were acquired to decipher the significant genes and pathways in FGF2-treated fibroblasts. Compared to microarrays, RNA-Seq technology shows higher sensitivity and increased quantitative accuracy and therefore detects even low abundance transcripts. Additionally, the RNA-Seq expands in terms of non-coding RNA detection (Schwingen et al. 2020). Here, we performed RNA-seq analysis for the dissection of the molecular profiles of FGF2-treated fibroblasts. Following the screening out the differentially expressed genes (DEGs), we identified the key genes and the signaling pathways triggered by FGF2 in skin fibroblasts. Thus, the study would provide a comprehensive understanding of the mechanisms regulated by FGF2 in skin fibroblasts, which may guide subsequent studies on skin wounds. 
112 MATERIALS AND METHODS

113 Cell cultures and reagents

114 Human skin biopsies were obtained from healthy subjects (Additional file 1). All the tissues

115 were collected in Dulbecco's modified Eagle's medium (DMEM; Gibco BRL, Grand Island, NY,

116 USA), and primary fibroblasts were established as described previously (Xuan et al. 2014).

117 Primary fibroblasts were grown in DMEM supplemented with 10\% fetal bovine serum (PAA

118 Laboratories, Etobicoke, Ontario, Canada), $100 \mathrm{U} / \mathrm{mL}$ penicillin, and $100 \mu \mathrm{g} / \mathrm{mL}$ streptomycin

119 (Gibco BRL) at $37^{\circ} \mathrm{C}$ in a humidified atmosphere containing 5\% CO2. When confluent, the cells 120 were trypsinized using a 0.25\% trypsin/0.02\% EDTA solution (Sigma, St Louis, MO, USA) and 121 subcultured at a 1:3 ratio on culture plastic dishes. After three or six passages, fibroblasts were 122 used for the following experiments. The human skin fibroblasts were cultured in serum-free 123 DMEM alone or DMEM with FGF2 (Proteintech, Rosemont, IL, Cat: HZ-1285) at varying doses $124(0,5,10$, and $50 \mathrm{ng} / \mathrm{ml})$ for $48 \mathrm{hrs}$. For RNA-seq assay, cells were treated with 10ng/ml FGF2.

\section{RNA isolation and RNA sequencing}

After 48 hours of culture, total RNA was extracted from 7 samples (4 samples from FGF2treated fibroblasts, 3 samples from control fibroblasts) using Trizol (Invitrogen, Carlsbad, CA). The libraries were constructed by RNA Library Prep Kit for Illumina (NEB, MA, and USA) according to the manufacturer's instructions. The library products were sequenced using Illumina HiSeqTM 2500 (Illumina, CA, USA). Index of the reference genome was built and paired-end clean reads were aligned to the reference genome using STAR. HTSeq v0.6.0 was used to count the reads numbers mapped to each gene. And then FPKM of each gene was calculated based on the length of the gene and reads count mapped to this gene. Differentially expressed RNA between the two groups was performed using the DESeq. 2R package (1.10.1). The resulting $\mathrm{p}$ values were adjusted using the Benjamini and Hochberg's approach. Genes with an adjusted $\mathrm{p}<$ 0.05 and $|\log 2 \mathrm{FC}|>1.0$ were assigned as differentially expressed genes (DEGs) or LncRNAs.

\section{Annotation for DEGs}

The DAVID Functional

Annotation

Bioinformatics

Microarray

Analysis 
141 (website:https://david.ncifcrf.gov/) was used for the GO enrichment and KEGG pathway analysis 142 (Xu et al. 2020). The GO Annotation for DEGs included three terms, molecular functions (MF), 143 biological processes (BP), and cellular components (CC) of genomic products. As for pathway 144 enrichment, DEGs were analyzed by the Kyoto encyclopedia of genes and genomes (KEGG) 145 pathway within the DAVID database (Version 6.7). $\mathrm{P}<0.05$ was considered as statistical significance for the correlations. The Bubble Charts were conducted using the ggplot2 package in R software.

\section{LncRNA-mRNA co-expression network construction}

To screen the significant lncRNAs, the LncRNA-TF network was constructed by the NetworkAnalyst website (https://www.networkanalyst.ca/). The parameters for the enrichment analysis were as follows. A specific organism was chosen H. sapiens (human). The ID type was chosen official gene symbol. TF-gene interaction was analyzed using the ENCODE database (Wang et al. 2020a). Based on the function of ECM organization in wound healing, the differentially expressed mRNAs involved in ECM organization were focused to analyze the associations between mRNAs and lncRNAs. The correlations of mRNAs and lncRNAs were calculated by the Pearson coefficients to construct the networks. The significant pairs of mRNAs-lncRNAs (coefficient $>0.95, \mathrm{P}<0.05$ ) were visualized by Cytoscape software.

\section{Quantitative real-time PCR}

Total RNA was isolated from tissues or cells by using TRIZOL reagent (Invitrogen, Carlsbad, CA) according to the manufacturer's protocol. First strand cDNA synthesis was performed on the total RNA $(0.5 \mu \mathrm{g})$ using the reverse transcription kit (Takara, Dalian, China). The real-time PCR assay was conducted with the SYBR Green PCR Kit (Takara, Dalian, China). The primers for target genes are listed in Additional file 2. The PCR reaction conditions were as follows: $95^{\circ} \mathrm{C}$ for $15 \mathrm{~s}$ followed by 40 cycles of $95^{\circ} \mathrm{C}$ for $5 \mathrm{~s}$, and $60^{\circ} \mathrm{C}$ for $30 \mathrm{~s}$. Real-time PCR results were performed by the $\Delta \Delta \mathrm{CT}$ method. 
169

170

171

172

173

174

175

176

177

178

179

180

181

182

183

184

185

186

187

188

189

190

191

192

193

194

195

196

\section{RESULTS}

\section{Transcriptional profiles in FGF2-treated fibroblasts.}

To determine the effects of FGF2 on the fibroblasts, skin fibroblasts were treated with FGF2 at different doses for 48 hours. As shown in Fig. 1A, morphological results from the microscope demonstrated skin fibroblasts revealed a smaller size, less spindle-like shape in a dose-dependent manner, indicating fibroblasts activation was suppressed by FGF2. FGF2 treatment at $10 \mathrm{ng} / \mathrm{ml}$ or $50 \mathrm{ng} / \mathrm{ml}$ significantly induced morphological changes in fibroblasts. Based on the FGF2 concentration $(4 \mathrm{ng} / \mathrm{ml})$ used in previous microarray studies (Hernandez \& Dominko 2016; Kashpur et al. 2013), skin fibroblasts were treated with FGF2 at $10 \mathrm{ng} / \mathrm{ml}$ for 48 hours and harvested for RNA-seq to understand the expression profiles in FGF2-treated fibroblasts. The RNA-seq result showed a total of 1475 mRNAs were differentially expressed in FGF2-treated fibroblasts (Fig.1B, Additional file 3), among which 676 genes were up-regulated and 799 genes were down-regulated (Fig.1C). Meanwhile, there were 213 LncRNAs with |fold change (FC) $\mid>2$ (Fig.1B, Additional file 4), among which 80 LncRNAs were up-regulated and 133 were down-regulated (Fig.1C).

\section{GO enrichment for DEGs.}

To explore the functions of the DEGs, the 1475 DEGs were annotated by three GO categories (biological processes, molecular functions, and cellular components). For GO biological process, DEGs were involved in the extracellular matrix (ECM) organization, angiogenesis, cell adhesion, positive regulation of cell migration, and collagen catabolic process (Fig.2, Additional file 5). For molecular function, the DEGs were significantly enriched in the regulation of calcium ion binding, growth factor activity, ECM structural constituent, heparin binding, and frizzled binding (Fig.2). For cellular component annotation, the most significant terms were enriched in extracellular space, extracellular region, proteinaceous ECM, ECM, and plasma membrane (Fig.2).

\section{KEGG pathway analysis.}


197

198

199

200

201

202

203

204

205

206

207

208

209

210

211

212

213

214

215

216

217

218

219

220

221

222

223

224

The KEGG pathway analysis was performed to identify the significant pathways induced by FGF2 in human skin fibroblasts. The 1475 DEGs were mapped to 44 KEGG pathways. Among these pathways, 37 pathways were significantly enriched $(\mathrm{P} \leq 0.05)$ (Additional file 6$)$. The top 10 significant pathways were represented, including ECM-receptor interaction, PI3K-Akt signaling pathway, Hippo signaling pathway, complement and coagulation cascades, TGF-beta signaling pathway, MAPK signaling pathway, proteoglycans in cancer, protein digestion, focal adhesion, and cell adhesion molecules (Fig.3).

\section{mRNA-IncRNA co-expression network.}

To investigate the regulatory networks of lncRNAs, the differentially expressed lncRNAs were uploaded within the NetworkAnalyst website. The PPI networks of 213 lncRNAs revealed the top 10 significant LncRNAs, including HOXA-AS2, LOC100130417, H19, LOC100507420, and SNHG8 (Fig. 4A). Based on the above result of GO enrichment, ECM organization was significantly involved in the FGF2-mediated biological process. There were 48 differentially expressed mRNAs involved in ECM organization. To explore associations between the 48 mRNAs and lncRNAs, pairs of mRNAs-lncRNAs were analyzed by the Pearson correlation analysis and were sorted by the coefficients, which represented the associations of mRNA and LncRNA in the networks (Additional file 7). As shown in Fig. 4B, the three key LncRNAs, including HOXA-AS2, H19, and SNHG8 were identified in the FGF2-mediated ECM organization. The three LncRNAs were significantly associated with six DEGs, including LOX, TGFB1, ITGA10, COL4A1, COL3A1, and PDGFA. Among the 6 DEGs, only ITGA10 was upregulated and the other 5 DEGs were downregulated (Fig. 4B).

\section{Validation of hub genes by real-time PCR}

To further verify the results of RNA-seq analysis, the levels of the six hub genes (COL3A1, COL4A1, LOX, PDGFA, TGFBI, and ITGA10) were validated. Firstly, the levels of six hub genes were compared between two GEO datasets (GSE60580 and GSE48967) and our RNA-seq data. As illustrated in Fig. 5A, our RNA sequencing data and the GEO datasets demonstrated 
225

226

227

228

229

230

231

232

233

234

235

236

237

238

239

240

241

242

243

244

245

246

247

248

249

250

ITGA10 was significantly upregulated while the other five genes were downregulated in FGF2treated fibroblasts. Next, the levels of six hub genes were validated by real-time PCR. As predicted by the above bioinformatics analysis, real-time PCR results also showed ITGA10 was significantly upregulated while the other five genes were downregulated in FGF2-treated fibroblasts (Fig. 5B).

\section{DISCUSSION}

Although FGF2 has been revealed to have the potential to attenuate fibrotic phenotypes and drive the more desirable, regenerative resolution of wound healing in damaged tissue, the mechanisms by which FGF2 ameliorates fibrosis are not entirely understood. As mentioned above, fibroblasts and myofibroblasts are the main effectors in the process of fibrosis. Of note, in vitro fibroblasts grown on two-dimensional (2-D) substrata flatten out and develop prominent stress fibers that are made of actin, myosin, and actin-binding proteins. This is in marked contrast with the in vivo situation, in which actively migrating fibroblasts do not contain stress fibers (Walpita \& Hay 2002). Thus, in vitro fibroblasts grown on tissue culture plastic or glass tend to reveal myofibroblast phenotypes. Consistent with the notion that in vitro fibroblasts are subject to be high stiffness, our study showed myofibroblast markers including ACTA2 were expressed in fibroblasts grown on plastic dishes. And the effects of FGF2 on transcriptomics of fibroblasts in the study may be more representative of the effects of pharmacologic exogenous FGF2 on myofibroblasts compared to the effects of endogenous FGF2 on quiescent fibroblasts. By the RNA-seq analysis, our study identified 1475 FGF2-responsive DEGs, including LOX, TGFB1, PDGFA, COL3A1, COL4A1, and ITGA10. Similar to our result, previous microarray assays were performed to identify the expression profile of FGF2-treated fibroblasts (Hernandez \& Dominko 2016; Kashpur et al. 2013). Based on the advantages of the RNA-Seq in terms of lncRNAs detection, our results further suggested HOXA-AS2, H19, and SNHG8 were involved in FGF2-mediated ECM organization. Therefore, the study would provide a comprehensive understanding of the FGF2-responsive genes in human skin fibroblasts, which may guide 
251

252

253

254

255

256

257

258

259

260

261

262

263

264

265

266

267

268

269

270

271

272

273

274

275

276

277

subsequent studies on wound healing.

Our first goal was to identify significantly differentially expressed genes (DEGs) in FGF2treated fibroblasts. Fibroblasts are responsible for ECM production during dermal wound healing. Here, our results showed that exogenous FGF2 affects a large number of genes involved in the production and remodeling of ECM. Type III collagen is a hallmark of several chronic fibrotic diseases including systemic sclerosis, cardiac fibrosis, lung fibrosis, liver cirrhosis, and renal fibrosis (Kuivaniemi \& Tromp 2019). Besides collagen III, FGF2 caused downregulation of other collagens such as collagen I, collagen V, collagen IV, collagen XI, and collagen XV, as well as caused upregulation of collagen X, collagen XIII, and collagen XVII (Additional file 3). The subsequent qRT-PCR analysis confirmed the downregulation of COL3A1 and COL4A1 in FGF2-treated fibroblasts (Fig.5B). In line with our result, the previous microarray study demonstrated COL3A1 and COL4A1 were downregulated by FGF2 in dermal fibroblasts(Kashpur et al. 2013). Besides the collagen, our result revealed other ECM genes affected by FGF2 treatment including laminins and fibronectins (Additional file 3). Similarly, FGF2 has previously been shown to significantly downregulated laminin alpha 2 (LAMA2) and Fibronectin 1 (FN1)(Kashpur et al. 2013). Thus, our study, together with previous observations, indicated FGF2 modulated the production of the ECM in human fibroblasts, which potentially favored the changes in cell attachment to ECM. Cell attachment to the ECM is regulated through integrins. Integrins constitute a subset of the integrin family with the affinity for GFOGER-like sequences in collagens and are crucial for dynamic connective tissue remodeling events--such as wound healing(Zeltz \& Gullberg 2016). Here, our results demonstrated FGF2 caused upregulation of numerous integrins such as integrin alpha 10 (ITGA10), integrin alpha 2, and integrin alpha 6, as well as caused downregulation of integrin beta like 1, integrin beta 8, and integrin beta 4 (Additional file 3). Most profoundly affected by FGF2 treatment was ITGA10. The qRT-PCR analysis confirmed the upregulation of ITGA10 (Fig.5B). ITGA10 was originally identified as a type II collagen-binding receptor on chondrocytes and mainly confined to cartilage-containing tissues(Camper et al. 2001). ITGA10 
278

279

280

281

282

283

284

285

286

287

288

289

290

291

292

293

294

295

296

297

298

299

300

301

302

303

was also observed to b up-regulated in malignant melanoma cells. And further investigation showed downregulating ITGA10 expression by an inhibitory antibody or an antisense construct had hindered migratory potential, suggesting a role for ITGA10 in melanoma cell migration(Wenke et al. 2007). Here, our study showed that exogenous FGF2 significantly induced the expression of ITGA10. Consistent with our result, the induce of ITGA10 expression by FGF2 previously occurred in mesenchymal stem cells and dermal fibroblasts(Kashpur et al. 2013; Varas et al. 2007). Thus, our study, together with previous observations, suggested FGF2 modulated the ITGA10 expression in human fibroblasts, which favored the migratory potential.

Following the identification of DEGs, GO analysis was performed to identify the important biological processes in human FGF2-treated fibroblasts. Our GO analysis demonstrated that the FGF2-associated DEGs were mainly enriched in the ECM organization, cell adhesion, and cell migration. This result accorded with the knowledge that FGF2 functioned as an important regulator in cell behavior, cell growth, and survival (Akl et al. 2016; Klagsbrun 1992; Przybylski 2009). Also, we further explored the effect of FGF2 on signal pathways in skin fibroblasts. KEGG enrichment results implied that FGF2 was mainly involved in classical pathways including ECM-receptor interaction and PI3K-Akt signaling pathway. Cell interactions with the ECM are mediated by integrins and various signaling cascades are activated, which control cell adhesion, proliferation, morphogenesis, differentiation, and survival (DiPersio \& Van De Water 2019). Of note, our enrichment results indicated that FGF2 was significantly associated with the Hippo signaling pathway. In line with our results, FGF2 was observed to promote the Hippo/YAP-signaling by inducing the nuclear-YAP expression during lens cell proliferation and differentiation, indicating FGF2 plays important roles in mediating the Hippo signaling pathway (Dawes et al. 2018). Furthermore, FGFR1 and FGFR2 were showed to directly interact with YAP/TAZ at multiple tyrosine residues independent of upstream Hippo signaling (Azad et al. 2020). Thus, our study, together with previous observations, suggested that the pivotal role of the FGF/FGFR signaling in mediating the Hippo signaling pathway. 
304

305

306

307

308

309

310

311

312

313

314

315

316

317

318

319

320

321

322

323

324

325

326

327

328

329

330

In addition to coding genes, this present study was focused on the differentially expressed LncRNAs in FGF2-treated fibroblasts. LncRNAs have been linked to the biological processes in various skin cells both physiological and pathological conditions, Although the role of lncRNAs in normal skin wound healing remains unexplored, emerging observations have linked lncRNAs to pathological scars. By microarray analysis, more than 2,500 lncRNAs were differentially expressed in keloid tissue compared with the normal human skin (Liang et al. 2015). Here, our study has revealed 213 differentially expressed LncRNAs and highlighted the three key LncRNAs (HOXA-AS2, H19, and SNHG8) in FGF2-treated fibroblasts. LncRNA H19, as a 2.3 $\mathrm{kb}$ IncRNA, is encoded from paternally imprinted and maternally expressed on human chromosome 11p15.5. The imprinted H19 is highly expressed in embryogenesis but is barely detectable in most tissues shortly after birth (Lustig et al. 1994). Numerous studies have revealed aberrant alterations of H19 expression in various tumors, implicating a crucial role of H19 in tumorgenesis (Ghafouri-Fard et al. 2020). Recently, H19 has also been observed to be upregulated in keloid tissues and fibroblasts. Moreover, silencing of H19 promoted cell viability, migration, and invasion of the fibroblasts(Wang et al. 2020b). In the present study, H19 was downregulated in the dermal fibroblast exposure to FGF2, suggesting FGF2 attenuated the expression of H19. Conversely, Sun et al reported the H19 levels were remarkably increased in FGF2-treated human umbilical vein endothelial cells (Sun et al. 2019). Thus, our study indicated the FGF2-mediated H19 expression appeared to exhibit a context-dependent pattern. In addition to H19, another two LncRNA HOXA-AS2 and SNHG8 were identified in FGF2-treated fibroblasts. Although numerous observations have demonstrated HOXA-AS2 and SNHG8 play vital roles in the development of various cancer including non-small cell lung cancer and breast cancer, gastric cancer (Chen et al. 2018; Wang et al. 2018). But, whether HOXA-AS2 and SNHG8 exhibit a certain function in fibroblasts remains elusive. Here, our result showed HOXA-AS2 and SNHG8 were downregulated in FGF2-treated fibroblasts. Taking into account the significance of FGF2 in skin wound healing, we conjectured that the novel lncRNAs (H19, HOXA-AS2. and SNHG8) may provide candidate mechanisms that may account for FGF2- 
331

332

333

334

335

336

337

338

339

340

341

342

343

344

345

346

347

348

349

350

351

352

353

354

355

356

mediated wound healing.

\section{CONCLUSIONS}

In summary, the current study carried out the RNA-seq analysis to identify the crucial genes in FGF2-treated skin fibroblasts. Our results showed FGF2 was associated with ECM organization as well as other biological processes including cell adhesion and cell migration. Furthermore, our study identified the key genes (LOX, TGFB1, PDGFA, COL3A1, COL4A1, and ITGA10), with ITGA10 being particularly prominent. Notably, our study highlighted the three key lncRNAs (HOXA-AS2, H19, and SNHG8) in FGF2-treated fibroblasts. Further studies are needed to delineate the mechanism that underlies the key LncRNAs in FGF2-mediated cellular functions. Therefore, the present study may provide new ideas and targets for the diagnosis and treatment of skin wound healing.

\section{ACKNOWLEDGEMENTS}

The authors gratefully thank Zi-Qi Zhou for his statistical assistance.

\section{ADDITIONAL INFORMATION AND DECLARATIONS}

\section{Funding}

This work was supported by the Fund from the National Natural Science Foundation of China (No.81401616) and the Natural Science Foundation of Shanghai (14ZR1405100).

\section{Declaration of interests}

The authors declare that they have no competing interests

\section{Authors' contributions}

Baojin Wu, Xinjie Tang and Ronghu Ke conceived and designed the study. Xiejie Tang and Baojin Wu analyzed the datasets and PCR validation. Zhaoping Zhou and Xinjie Tang performed bioinformatics analysis. Honglin $\mathrm{Ke}$ and Shao Tang contributed analysis tools. Ronghu Ke 
357

358

359

360

361

362

363

364

365

366

prepared the manuscript.

\section{Availability of data and materials}

Supporting data can be accessed if the corresponding author agrees.

\section{References}

Akita S, Akino K, Imaizumi T, and Hirano A. 2008. Basic fibroblast growth factor accelerates and improves second-degree burn wound healing. Wound Repair Regen 16:635-641. 10.1111/j.1524-475X.2008.00414.X

Akl MR, Nagpal P, Ayoub NM, Tai B, Prabhu SA, Capac CM, Gliksman M, Goy A, and Suh KS. 2016. Molecular and clinical significance of fibroblast growth factor 2 (FGF2 /bFGF) in malignancies of solid and hematological cancers for personalized therapies. Oncotarget 7:44735-44762. 10.18632/oncotarget.8203

Azad T, Nouri K, Janse van Rensburg HJ, Maritan SM, Wu L, Hao Y, Montminy T, Yu J, Khanal P, Mulligan LM, and Yang X. 2020. A gain-of-functional screen identifies the Hippo pathway as a central mediator of receptor tyrosine kinases during tumorigenesis. Oncogene 39:334-355. 10.1038/s41388-019-0988-y

Camper L, Holmvall K, Wangnerud C, Aszodi A, and Lundgren-Akerlund E. 2001. Distribution of the collagen-binding integrin alpha10beta1 during mouse development. Cell Tissue Res 306:107-116. 10.1007/s004410100385

Chen C, Zhang Z, Li J, and Sun Y. 2018. SNHG8 is identified as a key regulator in non-smallcell lung cancer progression sponging to miR-542-3p by targeting CCND1/CDK6. Onco Targets Ther 11:6081-6090. 10.2147/OTT.S170482

Dawes LJ, Shelley EJ, McAvoy JW, and Lovicu FJ. 2018. A role for Hippo/YAP-signaling in FGF-induced lens epithelial cell proliferation and fibre differentiation. Exp Eye Res 169:122-133. 10.1016/j.exer.2018.01.014

DiPersio CM, and Van De Water L. 2019. Integrin Regulation of CAF Differentiation and Function. Cancers (Basel) 11. 10.3390/cancers11050715

Dolivo DM, Larson SA, and Dominko T. 2017a. FGF2-mediated attenuation of myofibroblast activation is modulated by distinct MAPK signaling pathways in human dermal fibroblasts. J Dermatol Sci 88:339-348. 10.1016/j.jdermsci.2017.08.013 
388

389

390

391

392

393

394

395

396

397

398

399

400

401

402

403

404

405

406

407

408

409

410

411

412

413

414

415

416

417

418

419

Dolivo DM, Larson SA, and Dominko T. 2017b. Fibroblast Growth Factor 2 as an Antifibrotic: Antagonism of Myofibroblast Differentiation and Suppression of Pro-Fibrotic Gene Expression. Cytokine Growth Factor Rev 38:49-58. 10.1016/j.cytogfr.2017.09.003

Ghafouri-Fard S, Esmaeili M, and Taheri M. 2020. H19 lncRNA: Roles in tumorigenesis. Biomed Pharmacother 123:109774. 10.1016/j.biopha.2019.109774

Hernandez S, and Dominko T. 2016. Novel Protein Arginine Methyltransferase 8 Isoform Is Essential for Cell Proliferation. J Cell Biochem 117:2056-2066. 10.1002/jcb.25508

Kashpur O, LaPointe D, Ambady S, Ryder EF, and Dominko T. 2013. FGF2-induced effects on transcriptome associated with regeneration competence in adult human fibroblasts. $B M C$ Genomics 14:656. 10.1186/1471-2164-14-656

Klagsbrun M. 1992. Mediators of angiogenesis: the biological significance of basic fibroblast growth factor (bFGF)-heparin and heparan sulfate interactions. Semin Cancer Biol 3:8187.

Kuivaniemi H, and Tromp G. 2019. Type III collagen (COL3A1): Gene and protein structure, tissue distribution, and associated diseases. Gene 707:151-171. 10.1016/j.gene.2019.05.003

Liang X, Ma L, Long X, and Wang X. 2015. LncRNA expression profiles and validation in keloid and normal skin tissue. Int J Oncol 47:1829-1838. 10.3892/ijo.2015.3177

Lustig O, Ariel I, Ilan J, Lev-Lehman E, De-Groot N, and Hochberg A. 1994. Expression of the imprinted gene $\mathrm{H} 19$ in the human fetus. Mol Reprod Dev 38:239-246. 10.1002/mrd.1080380302

Matsumine H. 2015. Treatment of skin avulsion injuries with basic fibroblast growth factor. Plast Reconstr Surg Glob Open 3:e371. 10.1097/GOX.0000000000000341

Mery B, Vallard A, Rowinski E, and Magne N. 2019. High-throughput sequencing in clinical oncology: from past to present. Swiss Med Wkly 149:w20057. 10.4414/smw.2019.20057

Nunes QM, Li Y, Sun C, Kinnunen TK, and Fernig DG. 2016. Fibroblast growth factors as tissue repair and regeneration therapeutics. PeerJ 4:e1535. 10.7717/peerj.1535

Ono I, Akasaka Y, Kikuchi R, Sakemoto A, Kamiya T, Yamashita T, and Jimbow K. 2007. Basic fibroblast growth factor reduces scar formation in acute incisional wounds. Wound Repair Regen 15:617-623. 10.1111/j.1524-475X.2007.00293.x

Ornitz DM, Xu J, Colvin JS, McEwen DG, MacArthur CA, Coulier F, Gao G, and Goldfarb M. 1996. Receptor specificity of the fibroblast growth factor family. J Biol Chem 271:15292- 
15297. 10.1074/jbc.271.25.15292

Przybylski M. 2009. A review of the current research on the role of bFGF and VEGF in angiogenesis. J Wound Care 18:516-519. 10.12968/jowc.2009.18.12.45609

Schwingen J, Kaplan M, and Kurschus FC. 2020. Review-Current Concepts in Inflammatory Skin Diseases Evolved by Transcriptome Analysis: In-Depth Analysis of Atopic Dermatitis and Psoriasis. Int J Mol Sci 21. 10.3390/ijms21030699

Sun B, Ding Y, Jin X, Xu S, and Zhang H. 2019. Long non-coding RNA H19 promotes corneal neovascularization by targeting microRNA-29c. Biosci Rep 39. 10.1042/BSR20182394

Vallee A, and Lecarpentier Y. 2019. TGF-beta in fibrosis by acting as a conductor for contractile properties of myofibroblasts. Cell Biosci 9:98. 10.1186/s13578-019-0362-3

Varas L, Ohlsson LB, Honeth G, Olsson A, Bengtsson T, Wiberg C, Bockermann R, Jarnum S, Richter J, Pennington D, Johnstone B, Lundgren-Akerlund E, and Kjellman C. 2007. Alpha10 integrin expression is up-regulated on fibroblast growth factor-2-treated mesenchymal stem cells with improved chondrogenic differentiation potential. Stem Cells Dev 16:965-978. 10.1089/scd.2007.0049

Walpita D, and Hay E. 2002. Studying actin-dependent processes in tissue culture. Nat Rev Mol Cell Biol 3:137-141. 10.1038/nrm727

Wang J, Su Z, Lu S, Fu W, Liu Z, Jiang X, and Tai S. 2018. LncRNA HOXA-AS2 and its molecular mechanisms in human cancer. Clin Chim Acta 485:229-233. 10.1016/j.cca.2018.07.004

Wang J, Yu H, Yili A, Gao Y, Hao L, Aisa HA, and Liu S. 2020a. Identification of hub genes and potential molecular mechanisms of chickpea isoflavones on MCF-7 breast cancer cells by integrated bioinformatics analysis. Ann Transl Med 8:86. 10.21037/atm.2019.12.141

Wang Z, Feng C, Song K, Qi Z, Huang W, and Wang Y. 2020b. IncRNA-H19/miR-29a axis affected the viability and apoptosis of keloid fibroblasts through acting upon COL1A1 signaling. J Cell Biochem. 10.1002/jcb.29649

Wenke AK, Kjellman C, Lundgren-Akerlund E, Uhlmann C, Haass NK, Herlyn M, and Bosserhoff AK. 2007. Expression of integrin alpha10 is induced in malignant melanoma. Cell Oncol 29:373-386. 10.1155/2007/601497

Xu BF, Liu R, Huang CX, He BS, Li GY, Sun HS, Feng ZP, and Bao MH. 2020. Identification of key genes in ruptured atherosclerotic plaques by weighted gene correlation network 
452

453

454

455

456

457

458

459

460

461

462

463

analysis. Sci Rep 10:10847. 10.1038/s41598-020-67114-2

Xuan YH, Huang BB, Tian HS, Chi LS, Duan YM, Wang X, Zhu ZX, Cai WH, Zhu YT, Wei TM, Ye HB, Cong WT, and Jin LT. 2014. High-glucose inhibits human fibroblast cell migration in wound healing via repression of bFGF-regulating JNK phosphorylation. PLoS One 9:e108182. 10.1371/journal.pone.0108182

Yazdani S, Bansal R, and Prakash J. 2017. Drug targeting to myofibroblasts: Implications for fibrosis and cancer. Adv Drug Deliv Rev 121:101-116. 10.1016/j.addr.2017.07.010

Zeltz C, and Gullberg D. 2016. The integrin-collagen connection - a glue for tissue repair? J Cell Sci 129:1284. 10.1242/jcs. 188672 
Figure 1

Figure 1. Transcriptional profiles in skin fibroblasts treated with FGF2.

(A). Representative morphology of skin fibroblasts treated with FGF2 at indicated dose. Scale bar=50 $\mu \mathrm{m}$. (B). volcano plot of mRNAs (Left) and LncRNAs (Right) expression profiles. (C). Summary of differently expressed mRNAs and IncRNAs $(P<0.05$ and $|\log 2 \mathrm{FC}|>1.0)$.

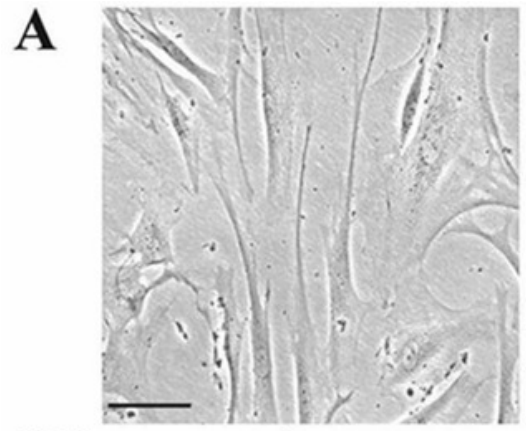

FGF2

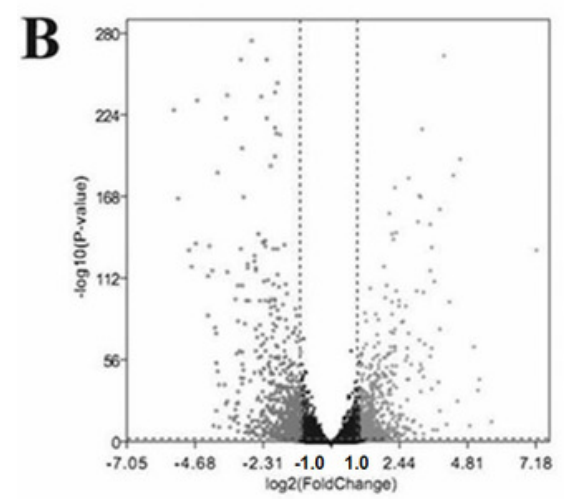

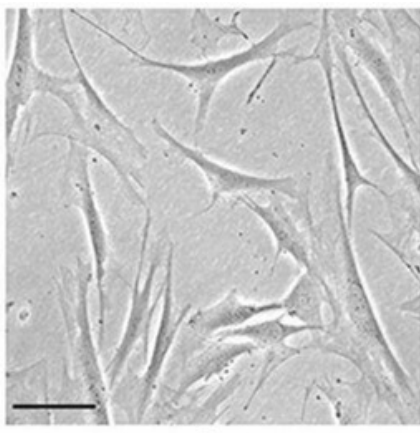

1

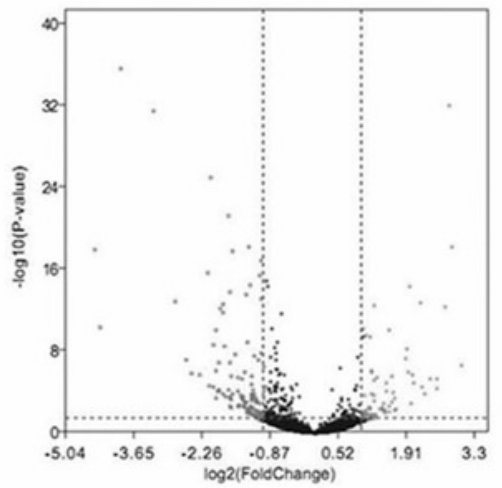

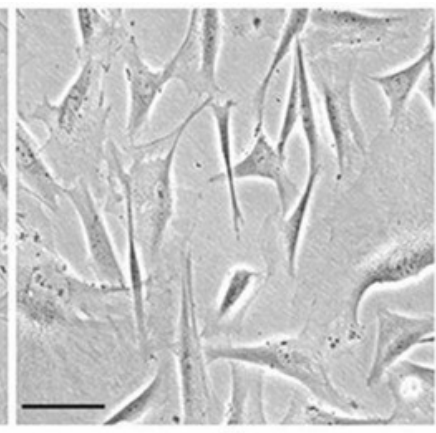

10

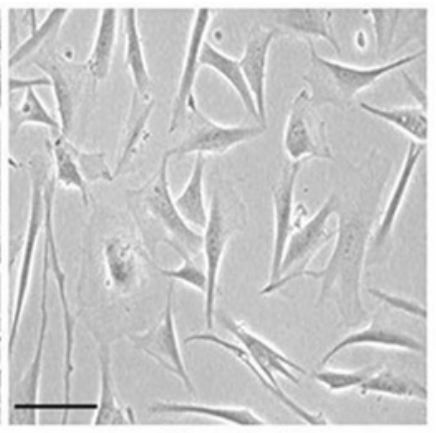

$50 \mathrm{ng} / \mathrm{mL}$

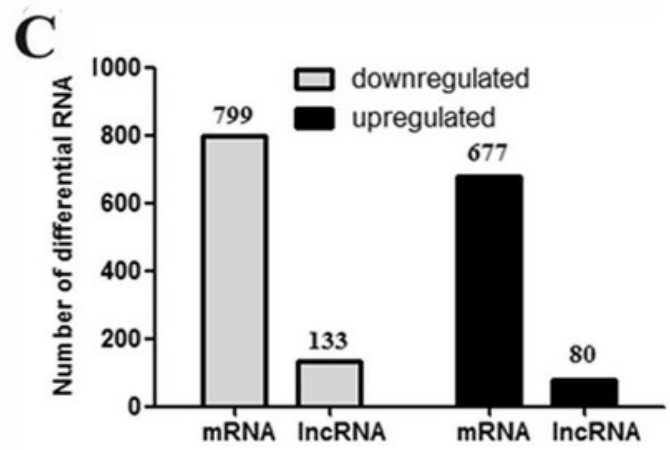


Figure 2

Figure 2. GO enrichment analysis of differently expressed genes (DEGs) in skin fibroblasts treated with FGF2.

The bar chart visualizes the top five significantly enriched items that are grouped by molecular functions, cellular component and biological process. The $\mathrm{x}$-axis indicates the term of GO enrichment, and Y-axis represents the number of hub genes enriched in a certain term.

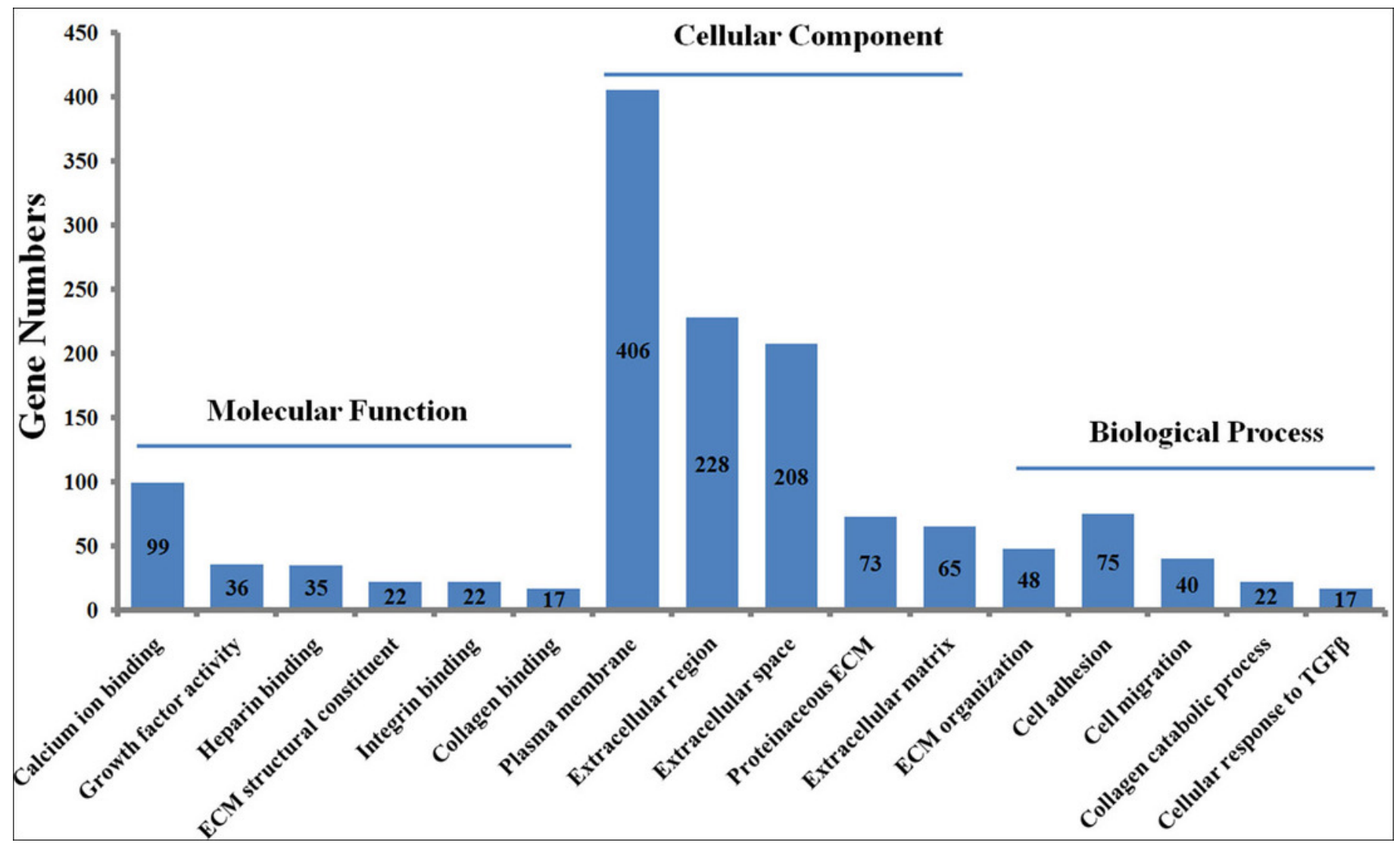


Figure 3

Figure 3. KEGG pathway analysis of differently expressed genes (DEGs) in skin fibroblasts treated with FGF2.

The bubble diagramrepresentsthe top 10 significantly enriched pathway. The $\mathrm{x}$ and $\mathrm{y}$ axis indicate the P value and pathways, respectively. Dot size denotes the count of enriched genes and dot color indicates $P$ value.

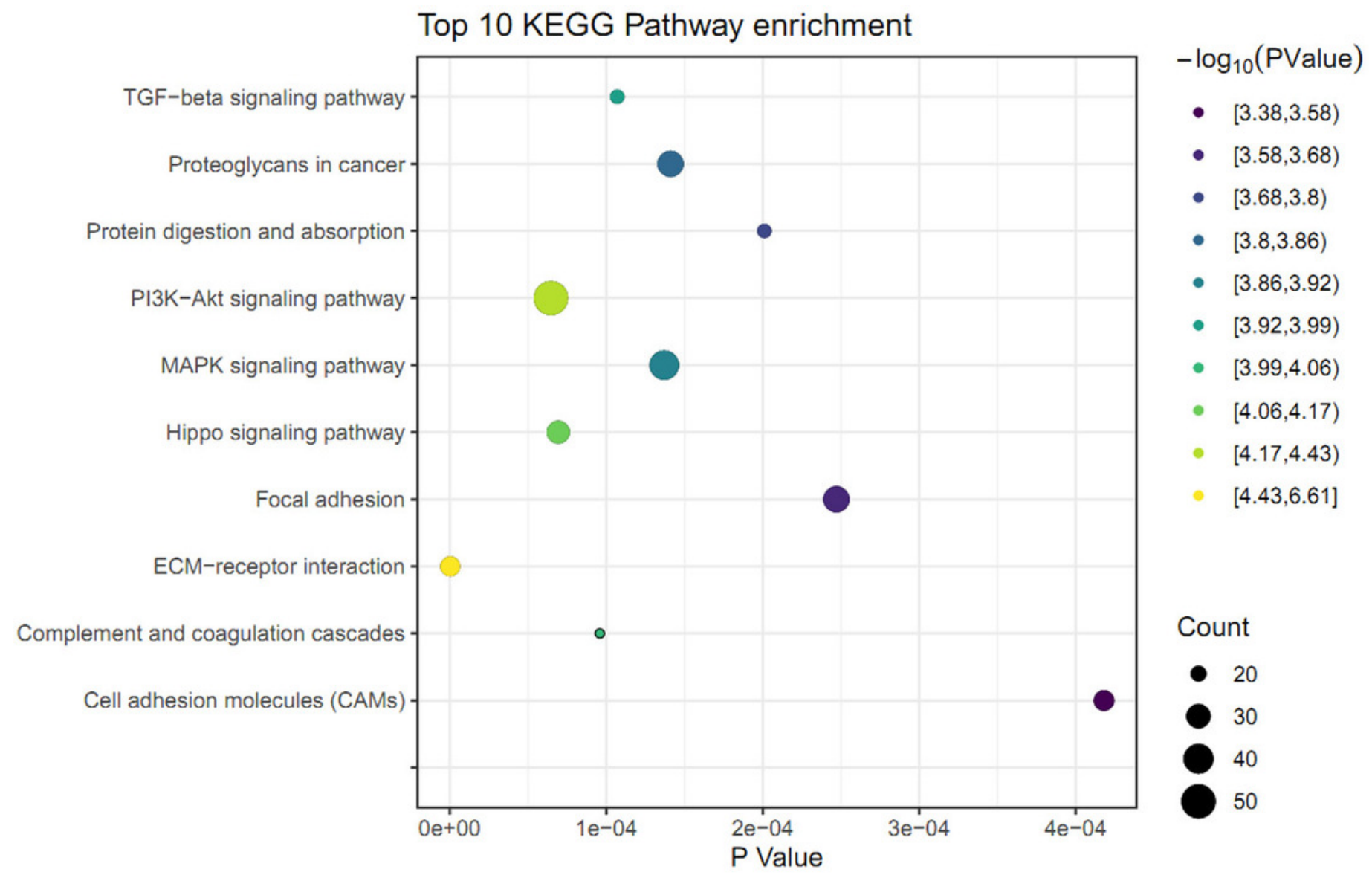




\section{Figure 4}

Figure 4. Networks of protein-protein interaction.

(A). Networks of the differently expressed LncRNAs created by the NetworkAnalyst website.

(B). The co-expression network of the ECM-associated mRNAs-IncRNAs created by Pearson's correlation. The diamond and ellipses nodes denote significant LncRNAs and mRNAs, respectively. The yellow and black nodes indicate upregulated and downregulated transcripts, respectively. 

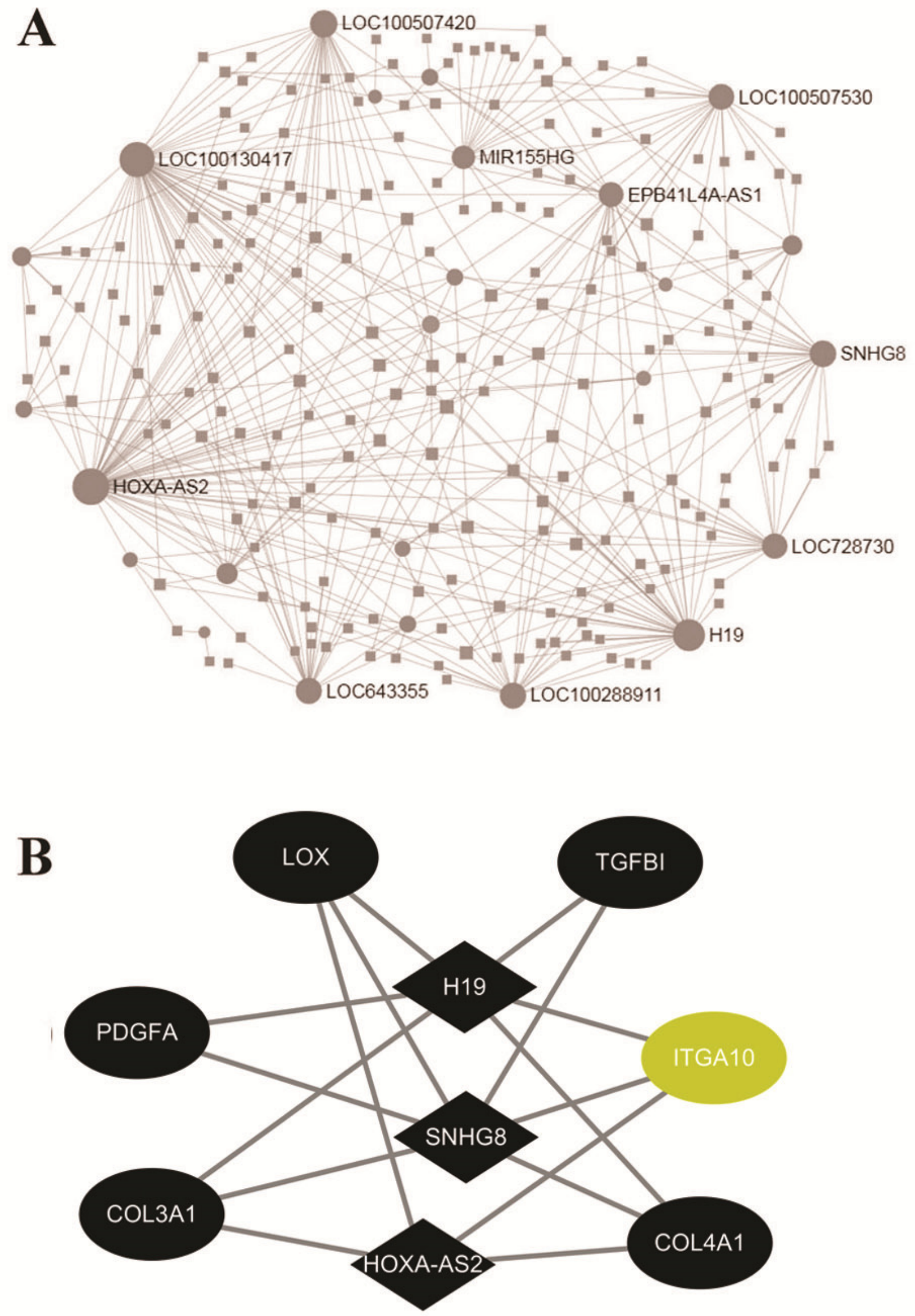


\section{Figure 5}

Figure 5.Validation of the expression for select genes.

(A). The expression levels of 7 genes in previous GEO microarray datasets and the present RNA-seq data. (B). The validation of 7 DEGs by real-time PCR. The $x$-axis shows DEGs and $y$ axis shows $\log 2$ (FoldChange). The log2(FoldChange) $>0$ and $\log 2$ (FoldChange) $<0$ indicate upregulation and downregulation, respectively. Statistical significance was assessed by Student's t-test. $* \mathrm{P}<0.05, * * \mathrm{P}<0.01$. 

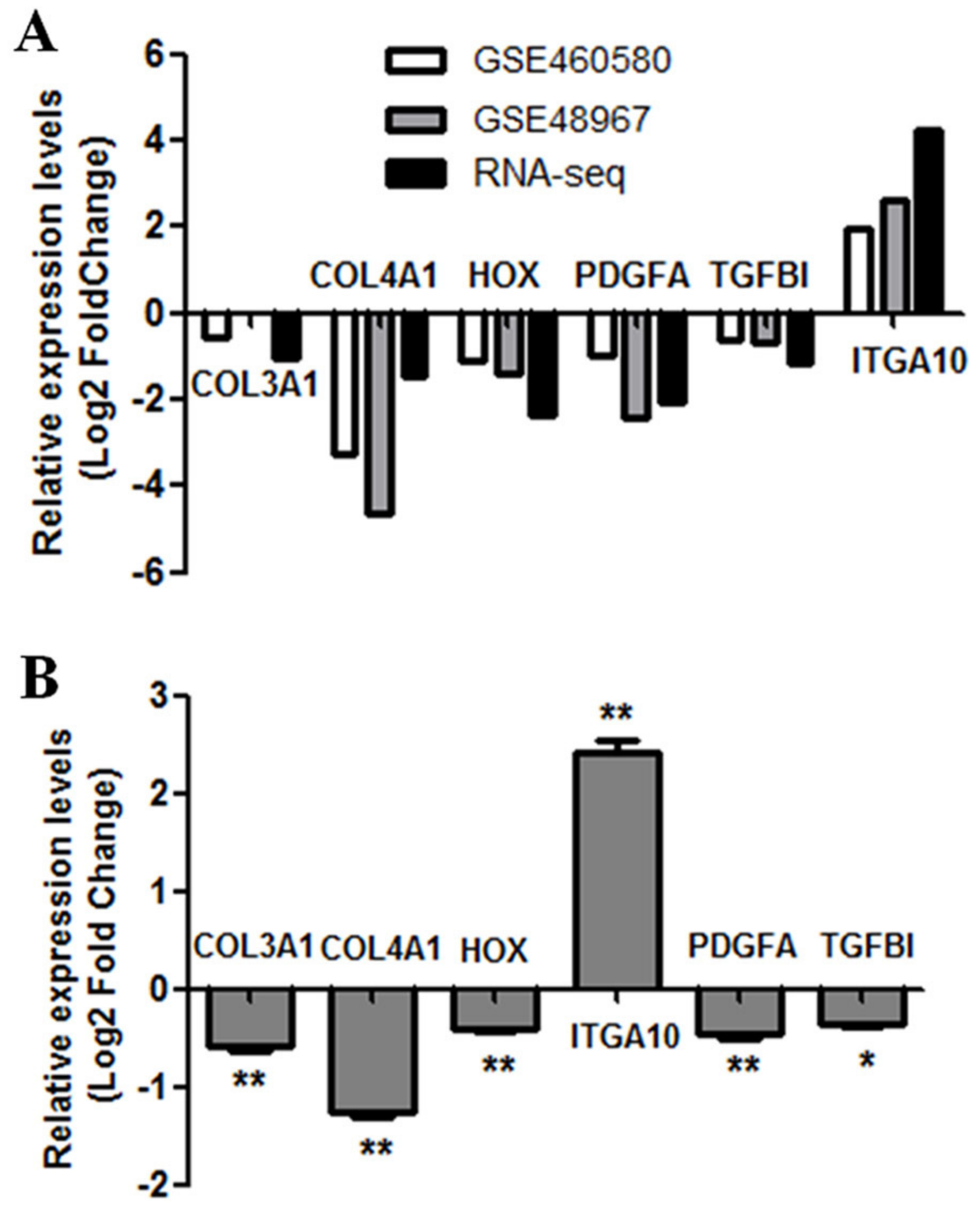